\title{
Diseño COMPUTACIONAL DE LA ESTRUCTURA DE UN BALANCE SOCIAL EN LAS ORGANIZACIONES DE ECONOMÍA SOLIDARIA
}

\author{
Computational design of the structure of a Social \\ BALANCE IN THE SOlidarity ECONOMY ORganizations
}

\author{
Nancy Edith Ochoa G1, Javier Ríos-Suárez ${ }^{2}$, Oscar Geovanni Hernández ${ }^{3}$, Lizeth Lucia Soler ${ }^{4}$ \\ Corporación Universitaria Minuto de Dios, Bogotá, Colombia.
}

Recibido: 13/02/2018 • Aprobado: 14/03/2018

\section{RESUMEN}

Este artículo muestra los resultados del proyecto de investigación "Diseño ingenieril del balance social para las organizaciones de economía solidaria”, su enfoque fue hacia la construcción de un modelo ingenieril que permitiera, a través de un aplicativo de escritorio, diseñar la primera etapa de un balance social para las organizaciones de economía solidaria en Colombia. Se basa en estrategias que a través de un sistema de cómputo logren facilitar la creación un balance social que facilite a las organizaciones tomar decisiones de acuerdo a los resultados obtenidos en los diferentes aspectos económicos, sociales, culturales, ambientales, administrativos, normativos y políticos, entre otros, de una organización, mediante el uso de indicadores cualitativos y cuantitativos, que abarcan estas siete (7) dimensiones. La metodología aplicada a la investigación es mixta (cuantitativa/cualitativa), debido a que para la recolección de los datos se contó con un modelo tipo "maqueta" en Excel con variables de tipo numérico (estadística) y de percepción (opinión). La estructura de la "maqueta" permitió generar un modelo ingenieril, para no solo suplir las necesidades inmediatas del cliente, sino a la vez crear procedimientos adecuados para soluciones similares. Por otro lado, el desarrollo del aplicativo se realizó bajo el marco ScrumBan y el modelo de desarrollo espiral.

Palabras clave: balance, indicadores, maqueta, solidario, scrumban.

\section{Abstract}

This article shows the results of the research project "Engineering design of social balance for Solidarity economy" ts approach was towards the construction of a engineeringmodel that allows, through an application of Desk, to design the first stage of a social balance for solidarity economyorganizations in Colombia. It is based on strategies that through a computer system facilitate the creation of a social balance that facilitates the organizations to ake decisions according

\footnotetext{
${ }^{1}$ nochoagueva@uniminuto.edu.co, orcid,org//0000-0002-4533-4990

2 jriossuarez@uniminuto.edu.co, orcid,org//0000-0001-8048-0590

3 chernal4@uniminuto.edu.co, orcid.org/0000-0002-3994-5269

${ }^{4}$ lsolersanch@uniminuto.edu.co, orcid.org/0000-0001-9147-3484
} 
to the results obtained in different environmental administrative, normative and political, among others, of an organization, through the use of qualitative and quantitative indicators cover these seven (7) dimensions

The methodology applied to the research is mixed (quantitativelqualitative), because for collection of data was counted a model type "mockup" in Excel with variables of numerical type (statistic) and perception (Opinion). The structure of the "model" allowed to generate a engineeringmodel, not only to meet the immediate needs of the client, but at the same time create appropriate procedures for similar solutions. On the other hand, the development of the application was carried out under the Scrumn framework and the spiral development model.

Key words: balance, indicators, model, scrumn.

\section{INTRODUCCIÓN}

Las empresas de economía solidaria además de la presentación de balances financieros, están obligadas a reportar indicadores de su gestión en el ámbito social según la ley 454 de 1998.

Los estamentos de la Organización Internacional del Trabajo (OIT), en el documento Manual de Balance Social (Balanced ScoreCard Institute,s.f.); sugieren un aproximado de 200 indicadores para generar el balance social, pero, para la regulación y unificación de las diferentes cooperativas es necesario conocer las leyes que regulan su funcionamiento, dándole un orden, lo que ayuda a generar confianza a los posibles asociados que contribuirán a que este sector siga su crecimiento normal.

Las empresas que conforman el sector solidario, se encontraban conformadas hasta el año 2015, por alrededor de 8.000 cooperativas, más de 3.127 fondos de empleados y 456 asociaciones mutuales. De acuerdo con las estadísticas de la Supersolidaria (entidad gubernamental que vigila a la economía solidaria en Colombia). Los estratos que más recurren a ellas son estratos socioeconómicos bajos (estratos 1, 2 y 3), ya que uno de sus atractivos son las bajas tasas de interés.

Estas organizaciones actualmente no cuentan con una estrategia adecuada para el diseño y control del estado de su balance social a través del esquema de indicadores como lo solicita la Superintendencia de Economía Solidarias en Colombia, según algunos estudios y lo reportado por los profesores Prada, Sierra y Mongui en el avance de su proyecto de investigación "Balances sociales para las economías solidarias" de la Facultad de Ciencias Empresariales en la Corporación Universitaria Minuto de Dios (Uniminuto). Donde estos indicadores deben precisarse en su valor, medición cualitativa y cuantitativa en el ámbito social, administrativo, económico, ambiental, cultural, político y normativo entre otros, tanto al exterior como al interior de dichas organizaciones, concertados bajo la estructura de plan estratégico debidamente consolidado.

Teniendo en cuenta lo anterior, en este trabajo se estableció un modelo computacional para contemplar todos los aspectos entregados y así generar un alcance más nutritivo en las organizaciones del sector solidario, logrando entregar resultados esperados con base en objetivos estratégicos, para que la evolución de las organizaciones de economía solidaria tenga un enfoque más productivo hacia lo social.

\section{MÉTodo}

El proyecto fue enfocado en una investigación de tecnología e innovación, ya que el propósito fue lograr hacer la transición de un modelo tipo maqueta de un 
balance social a un aplicativo de escritorio. Logrando innovar a través del uso de tecnología libre, sistematizando el conocimiento en un "nuevo producto" renovado en diseño y servicio, abriendo canales de competitividad en dichas organizaciones en el mercado nacional e internacional.

Por otra parte, el proyecto se basó en un esquema cualitativo y cuantitativo llamado también mixto, debido a que en su proceso de recolección de datos se analiza y vincula datos de tipo estadístico (numéricos) y de tipo percepción (opinión) en un mismo esquema, como es la medición numérica en las dimensiones social, cultural, política, normativa, administrativa, económica, financiera y ambiental entre otros, como por la percepción de algunas entidades con respecto al índice relativo que se alcanzó en esta triangulación de datos.

Lo cual responde al planteamiento y objetivos del estudio como lo es la "maqueta" del balance social para las organizaciones de economía solidaria (Salgado Levano, 2007).

Además de lo anterior se hizo uso de un tipo de estudio descriptivo, y para gestionar el proyecto se utilizó el marco Scrumban, que toma aspectos de la metodología Scrum y Kanban facilitando la ejecución de este marco de gestión. Por último, se utilizó el modelo espiral mediante el cual se realizó el desarrollo del aplicativo y se ejecutaron las diferentes etapas del ciclo de vida del mismo.

El producto de software se construyó en lenguaje Java SE, este cuenta con una pantalla de logeo, luego de acceder al aplicativo el usuario ve un menú con cuatro (4) opciones que permiten visualizar el alcance del mismos, las cuales son:

Indicadores: en el cual el usuario puede ingresar a una de las siete (7) dimensiones de los indicadores y hacer su medición acorde a los datos de ingreso. Allí se puede ver el resultado que arrojó este indicador, el porcentaje de cumplimiento mediante un objeto semáforo que cambia de color (verde cumplimiento del $90 \%$ al $100 \%$ de la meta, amarillo, cumplimiento del $70 \%$ al $89 \%$ de la meta, rojo cumplimiento del $50 \%$ al $69 \%$ y negro cumplimiento menor o igual al 49\%) y la variación entre la meta y el resultado obtenido (Alarcón \& Callejas, 2009).

Estado financiero: en esta opción el usuario puede registrar la información a nivel económico de la organización.

Planeación estratégica: allí el usuario puede registrar la información de los planes de mejora de acuerdo a los resultados obtenidos en la medición de los indicadores que realizó previamente. Además de generar una matriz DOFA y validar la competitividad de las demás organizaciones frente a esta, enlazar un objetivo al indicador que desea medir, entre otros.

Reportes/Informes: el usuario puede generar un reporte general, o por fecha de los indicadores, la planeación estratégica y la información financiera, el cual es exportado en un archivo plano.

\section{Resultados}

Se implementó el marco de gestión ScrumBan que permitió, generar una serie de actividades, y organizarlas de acuerdo a su prioridad, y responsable, con la finalidad de generar más tareas a medida de finalizar las existentes; todo ello contribuyó a la organización del desarrollo de la investigación. Además de ello y ya que este aplicativo tenia usuarios interesados, se creaban diferentes reuniones en las que se validaba el avance de las tareas y en las que el usuario podía dar su opinión y considerar junto a los investigadores un ajuste si lo veía necesario.

Por otro lado, se hizo uso del modelo espiral para llevar a cabo cada una de las fases del ciclo de vida del aplicativo. Con ello se obtuvieron requerimientos a nivel funcional y no funcional, los cuales fueron utilizados a la hora de evaluar la calidad y por ende la satisfacción del usuario final en la entrega del producto. 
A nivel técnico el aplicativo cuenta con una conexión a una base de datos relacional de MySql, la cual se encuentra normalizada hasta la cuarta (4) forma normal (FN), y está conformada por dieciocho (18) tablas, de las cuales tres (3) son parametrizadas, ya que contienen las siete (7) dimensiones de los indicadores como son: la dimensión social, cultural, política, normativa, administrativa, económica, y ambiental, los ciento veintiocho (128) indicadores, y los conceptos de financiera (Pérez, 2004; Faedei/Gizatea, 2012), cuenta, además, con un total de 160 formularios, en los cuales se abarcan todas las funcionalidades anteriormente mencionadas, en las cuales se utilizaron los siguientes objetos (GNU, s.f.):

- JFrame

- JLabel

- JTextField

- JComboBox

- Jtable

- JButtom

- JToolbar

- JDateSelected

- JTextArea

Por otro lado, se realizaron las siguientes pruebas en las cuales se validaron las funcionalidades del aplicativo (Hernández Rojas, 2012):

Pruebas de caja negra

- Prueba de situación de excepción Login.

- Prueba de valor límite mínimo indicador cualitativo (Interfaz mujeres cabeza de familia)

- Prueba de casos de uso reportes (Interfaz indicador)

- Prueba de casos de uso financiera (Interfaz balance)

- Prueba de situación de excepción Planeación estratégica (Interfaz cadena de valor)

- Pruebas de caja blanca

- Prueba de condición Login

- Prueba de expresiones lógicas indicador cualitativo

- Prueba de condición reportes (Interfaz indicador)

- Prueba de condición financiera (Interfaz balance)

- Prueba de condición planeación estratégica (Interfaz cadena de valor)
Además, como documentación para el aplicativo se elaboraron los manuales de usuario, manual técnico el cual contiene los diagramas de uso, de carril de natación, de clases, diccionario de datos, entidad relación, de secuencia y de flujo del aplicativo, y de instalación en el cual se explica el paso a paso del ejecutable.

A continuación, se visualiza en la figura 1 la pantalla principal del aplicativo donde se observan los cuatro módulos principales:

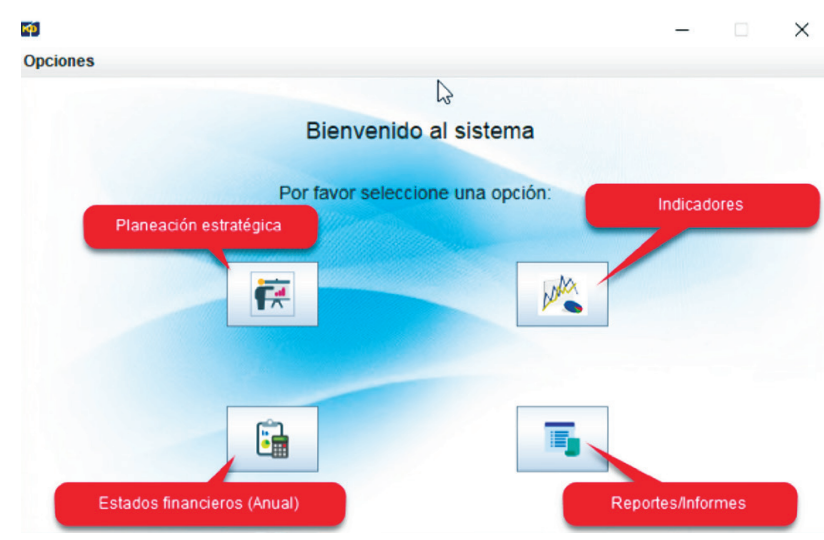

Fig. 1. Pantalla principal del aplicativo Fuente: autores

En la figura 2. se aprecia las dimensiones con las que se implementó la solución sistematizada como propuesta del balance social:

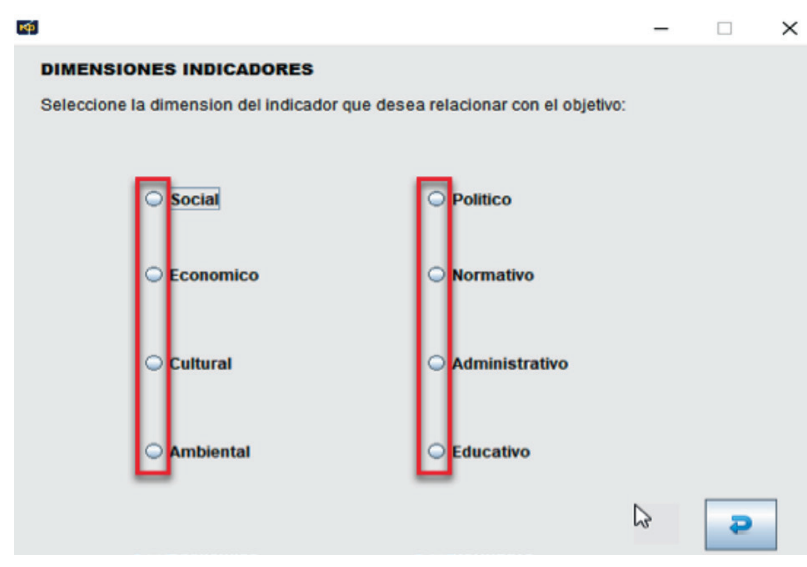

Fig. 2. Dimensiones del balance social

Fuente: autores 
Cada una de estas dimensiones contiene un grupo de indicadores, tal como se aprecia en la figura 3, cuando se selecciona la dimensión social se activan únicamente los indicadores respectivos y así sucesivamente con las demás.

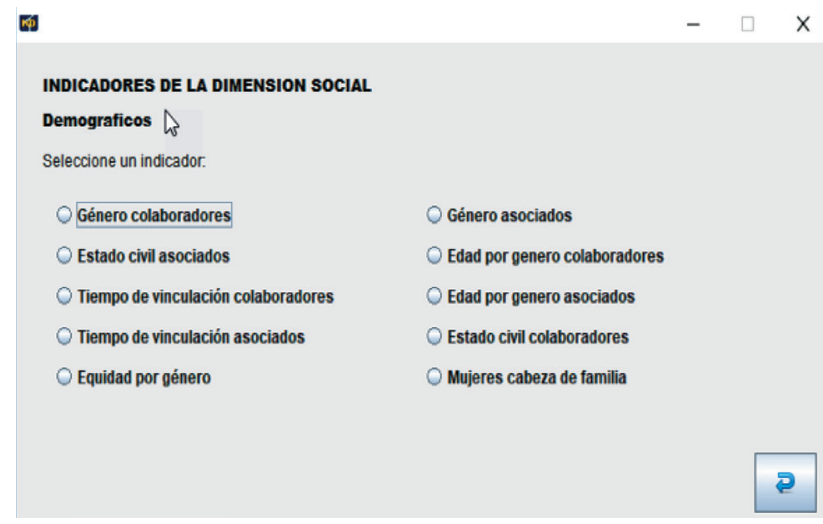

Fig. 3. Pantalla principal del aplicativo Fuente: autores

Si en la anterior figura se activa el indicador de Género de colaboradores, de inmediato muestra una pantalla con la estructura del formulario para recoger y operar los datos del indicador y así programar su estado en el espacio y tiempo directamente en el balance social para su seguimiento, cumplimiento y apoyo en el proceso tal como se aprecia e la figura 4.

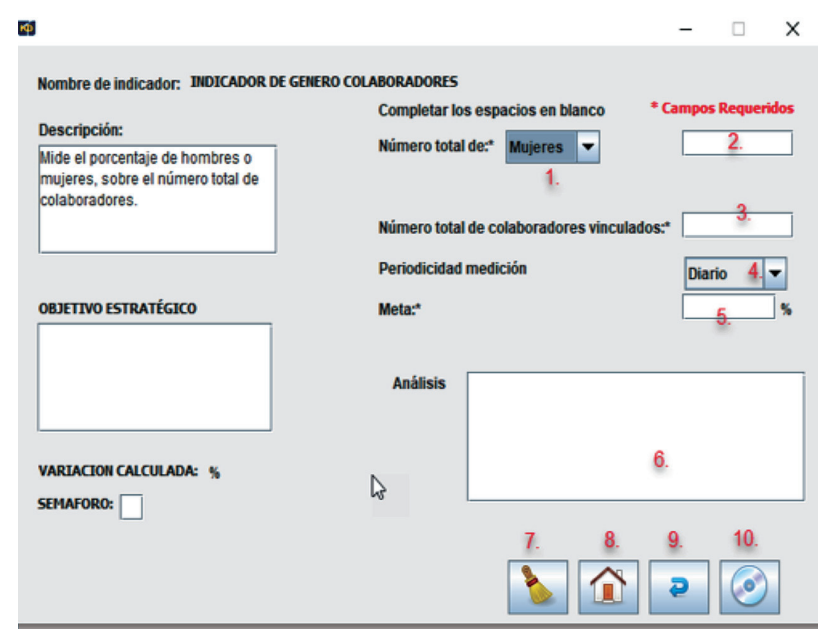

Fig. 4. Indicador de género de colaboradores Fuente: los autores
Algunos investigadores como Salgado, Callejas y Pérez han proporcionado una estructura para consolidar los indicadores cualitativos y cuantitativos de un balance social como apoyo a las organizaciones que se encuentran inscritas a la Superintendencia de Solidaridad en Colombia en el año 2014, bajo un esquema en Excel con el manejo de tablas dinámicas y objetivos específicos presentan una solución muy similar a la presentada en este proyecto.

Sin embargo, al transcurrir del tiempo se ha observado que esta solución presentada por los autores quedo demasiado corta ya que los indicadores tienden a crecer y a exigir una estructura en prospectiva para dicho balance.

Por tanto, presentar una propuesta de un sistema computacional con un aplicativo específico abierto, con el fin de ir atendiendo el crecimiento de estos indicadores en el espacio y tiempo, se ha considerado como una solución óptima la cual requiere ser fortalecida a través de la aplicación de estándares y/o normas de calidad del software, donde se logre construir un producto en línea a la medida de estas organizaciones, y, lo más importante que permita la toma de decisiones de forma inmediata y precisa.

Se espera que este estudio sea la base para trabajos futuros en desarrollo e innovación en la construcción ideal del alance social para las organizaciones que conforman la Súper Intendencia Solidaria en Colombia y así lograr una solución integral para todos.

\section{Conclusiones}

El identificar la estructura del modelo de la "maqueta” para el balance social, fue pieza clave para organizar el modelo ingenieril del aplicativo. Desde su frameworks Scrum, su esquema de base de datos con MySQLSB, el servidor de conexiones WampServer, el editor de código con el IDE_Netbeens y el lenguaje Java para escritorio (Pressman, 2013; Superintendencia de la Economía Solidaria (2017). 
Se logró la normalización e integridad de la base de datos, a través de su respectivo modelo entidad/relación y su diccionario de datos.

El uso del marco Scrum fue de gran utilidad ya que facilitó el control de las actividades en el desarrollo del proyecto, y al ser flexible se ajustó a las necesidades de tiempo de los integrantes del equipo, en el caso de reuniones presenciales.

El uso de formularios (JFrame) en java permitió que el desarrollo fuera más estructurado hacia una interfaz de fácil uso para el usuario y con ello se logró uno de los requerimientos solicitados por el cliente, que era lograr conseguir una interfaz amigable, de fácil acceso y sobre todo muy visible por sus objetos e imágenes (Whitepaper, s.f.).

\section{Agradecimientos}

Los autores agradecen especialmente a los investigadores Juan Salvador Prada, Javier Mongui y Alexandra Sierra pertenecientes a la Facultad de Ciencias Empresariales de la Corporación Universitaria Minuto de Dios por su valiosa colaboración y su gran aporte en la realización de este proyecto.

\section{REFERENCIAS}

Alarcón, A. \& Callejas, M. (2009). Propiedad intelectual y derechos de autor en el software libre. Revista Virtual Universidad Católica del Norte, 28, 1-16. Recuperado en: http://www.redalyc.org/articulo.oa?id=194214468009

Balance ScoreCard Institute. (s.f.) Generación de indicadores irrefutables. Recuperado en: http://www.balancedscorecard.org/ BSC-Basics/About-the-Balanced-Scorecard

Faedei / Gizatea. Software de Balance social (2012). Recuperado en: http://floss.esle.eu/search/?type_of_search=docs\&group_id $=81 \&$ words $=$ Balance + social $\&$ Search $=$ Busca

GNU. (s.f.). Software Libre. Recuperado de: https://www.gnu.org/ philosophy/free-sw.es.html

Hernández Rojas, D. D.H.R. (2012). Proyecto educativo social y empresarial P.E.S.E.M Recuperado en: http://www.ustacoopltda.com/Descargar.php?Descargable $=37$

Pérez Lamancha, B. (2006). Proceso de Testing Funcional e independiente. Recuperado en: http://www.ces.com.uy/documentos/imasd/Tesis-Beatriz_Perez_2006.pdf

Pressman, R. (2013). Ingeniería del Software: un enfoque práctico ( $7^{\mathrm{a}}$ edición). México D.F.: McGraw-Hill.

Salgado Levano, A. C. (2007), Investigación cualitativa: diseños, evaluación del rigor metodológico y retos. Recuperado de: http://www.scielo.org.pe/scielo.php?pid=S172948272007000100009\&script=sci_arttext\&tlng=en

Superintendencia de la Economía Solidaria. (2017). Instructivo reporte del formulario oficial de rendición de cuentas - SICSES. Recuperado en: http://www.supersolidaria.gov.co/sites/default/ files/public/sicses/d-gein- 010_instructivo_reporte_formulario_oficialrendicion_cuentas_-_sicses_v4.pdf

Whitepaper.(s.f.).Scrumvs.Kanbanvs.Scrumban.Recuperadoen:http:// www.eylean.com/Publications/DownloadPublication/4e93cecc4cb2-4e3f-849d-810d7aea33a5?name=Whitepaper---Scrum-vsKanban-vs-Scrumban. 